\title{
Cosmological Model with Interconnection between Dark Energy and Matter
}

\author{
Gennady S. Bisnovatyi-Kogan ${ }^{1,2}$ \\ 1 Space Research Institute of Russian Academy of Sciences, Profsoyuznaya 84/32, 117997 Moscow, Russia; \\ gkogan@iki.rssi.ru \\ 2 National Research Nuclear University MEPhI (Moscow Engineering Physics Institute), Kashirskoe Shosse 31, \\ 115409 Moscow, Russia
}

Citation: Bisnovatyi-Kogan, G.S. Cosmological Model with Interconnection between Dark Energy and Matter. Universe 2021, 7, 412. https: / / doi.org/10.3390/ universe7110412

Academic Editors: Galina L. Klimchitskaya, Vladimir M. Mostepanenko and Nazar R. Ikhsanov

Received: 11 October 2021

Accepted: 27 October 2021

Published: 29 October 2021

Publisher's Note: MDPI stays neutral with regard to jurisdictional claims in published maps and institutional affiliations.

Copyright: (C) 2021 by the author. Licensee MDPI, Basel, Switzerland. This article is an open access article distributed under the terms and conditions of the Creative Commons Attribution (CC BY) license (https:/ / creativecommons.org/licenses/by/ $4.0 /)$.

\begin{abstract}
It is accepted in the present cosmology model that the scalar field, which is responsible for the inflation stage in the early universe, transforms completely into matter, and the accelerated universe expansion is presently governed by dark energy (DE), whose origin is not connected with the inflationary scalar field. We suppose here that dark matter (DM) has a common origin with a small variable component of dark energy (DEV). We suggest that DE may presently have two components, one of which is the Einstein constant $\Lambda$, and another, smaller component DEV $\left(\Lambda_{V}\right)$ comes from the remnants of the scalar field responsible for inflation, which gave birth to the origin of presently existing matter. In this note we consider only the stages of the universe expansion after recombination, $z \simeq 1100$, when DM was the most abundant component of the matter, therefore we suggest for simplicity that a connection exists between DM and DEV so that the ratio of their densities remains constant over all the stages after recombination, $\rho_{D M}=\alpha \rho_{D E V}$, with a constant $\alpha$. One of the problems revealed recently in cosmology is a so-called Hubble tension (HT), which is the difference between values of the present Hubble constant, measured by observation of the universe at redshift $z \lesssim 1$, and by observations of a distant universe with CMB fluctuations originated at $z \sim 1100$. In this paper we suggest that this discrepancy may be explained by deviation of the cosmological expansion from a standard Lambda-CDM model of a flat universe, due to the action of an additional variable component DEV. Taking into account the influence of DEV on the universe's expansion, we find the value of $\alpha$ that could remove the HT problem. In order to maintain the almost constant DEV/DM energy density ratio during the time interval at $z<1100$, we suggest the existence of a wide mass DM particle distribution.
\end{abstract}

Keywords: dark energy; dark matter; Hubble constant

\section{Introduction}

During his long and successful scientific career, Yu. N. Gnedin worked also on problems on the border of physics and cosmology, namely on the possibility of direct searching, in astronomical observations, for axions, belonging to the family of Goldston bosons. These tiny particles, introduced by theoretical physicists, have been proposed as candidates for dark matter, whose presence was necessary for interpretation of different astronomical observations. During the years 1992-2009, Yu. N. Gnedin published at least six papers on this intriguing topic [1-6]. The cosmological model considered in this paper demands the presence of very light particles in DM, and axions could be the best candidates for that.

Here we consider a cosmological model that differs slightly from the widely accepted $\Lambda C D M$ model by having two components of the dark energy instead of the usually considered one component represented by $\Lambda$. The model considered here is constructed in order to explain the so called "Hubble Tension", which is the observed discrepancy between different averaged "local" measurements of the Hubble constant from one side, 
and distant measurements based on the analysis of the CMB fluctuations, connected with the recombination epoch at redshift $z \sim 1100$. In order to compare these two measurements, it was necessary to specify the cosmological model for determining the present value of the Hubble constant from distant measurements. We accept that the origin of HT is connected with the not fully appropriate $\Lambda C D M$ model, and suggest its modification removing this discrepancy.

While the present value of the Hubble constant $(\mathrm{HC})$ is one of the most important cosmological parameters, its measurements for many years have been performed by different astronomical groups ("local measurements"). These measurements have been based on several steps in order to have a possibility for independent distance measurements of more and more distant objects with observed runaway velocities. The use of different steps in this sequence by different groups has resulted in finding different values for the $\mathrm{HC}$, from $\sim 50 \mathrm{~km} / \mathrm{s} / \mathrm{Mps}$ by the Sandage-Tamman group to $\sim 100 \mathrm{~km} / \mathrm{s} / \mathrm{Mps}$ by the de Vacouleurs group [7]. Over time the local HC measurements at redshift $z \lesssim 1$ have been substantially improved due to construction of big telescopes and measurements by the Hubble mission, which permitted the narrowing of this interval of the $\mathrm{HC}$ to $72-75 \mathrm{~km} / \mathrm{s} / \mathrm{Mps}$.

The measurements of the CMB fluctuations by instruments in the satellites WMAP and PANCK gave the possibility for fully independent HC measurements at the recombination epoch. HT appears when you compare the present values of the HC obtained from CMB using a simple $\Lambda C D M$ model with local measurements. It is claimed that the discrepancy in these two values is statistically significant in the range $4.5 \sigma$ to $6.3 \sigma$ [8]; see, nevertheless [9].

It is accepted in the present cosmology model that the scalar field, which is responsible for the inflation stage in the early universe, transforms completely into matter, and the accelerated universe expansion is presently governed by dark energy (DE), whose origin is not connected with the inflationary scalar field. We suppose here that dark matter (DM) has a common origin with a small variable component of dark energy (DEV). We suggest that DE presently may have two components, one of which is the Einstein constant $\Lambda$, and another, smaller component DEV $\left(\Lambda_{V}\right)$ comes from the remnants of the scalar field responsible for inflation, which gave birth to the origin of presently existing matter. In this note we consider only the stages of the universe's expansion after recombination, $z \simeq 1100$, when DM is the most abundant component of the matter; therefore, we suggest for simplicity that a connection exists between DM and DEV so that the ratio of their densities remains constant over all the stages after recombination $\rho_{D M}=\alpha \rho_{D E V}$, with a constant $\alpha$.

In this paper we suggest that this discrepancy may be explained by the deviation of the cosmological expansion from a standard Lambda-CDM model of a flat universe, due to the action of an additional variable component DEV. Taking into account the influence of DEV on the universe's expansion, we find the value of $\alpha$ that could remove the HT problem. In order to maintain the almost constant DEV/DM energy density ratio during the time interval at $z<1100$, we suggest the existence of a wide mass DM particle distribution.

\section{Universe with Common Origin of DM and DE}

The scalar field with the potential $V(\phi)$, where $\phi$ is the intensity of the scalar field, is considered the main reason for the inflation [10,11], but see [12]. The equation for the scalar field in the expanding universe is written as [13]

$$
\ddot{\phi}+3 \frac{\dot{a}}{a} \dot{\phi}=-\frac{d V}{d \phi} .
$$

Here $a$ is a scale factor in the flat expanding universe [7]. The density $\rho_{V}$ and pressure $P_{V}$ of the scalar field ${ }^{1}$ are defined as [13]

$$
\rho_{V}=\frac{\dot{\phi}^{2}}{2}+V, \quad P_{V}=\frac{\dot{\phi}^{2}}{2}-V .
$$


Consider the universe with the initial scalar field, at initial intensity $\phi_{\text {in }}$ and initial potential $V_{i n}$, and at zero derivative $\dot{\phi}_{i n}=0$. The derivative of the scalar field intensity is growing on the initial stage of inflation.

Let us suggest that after reaching the relation

$$
\dot{\phi}^{2}=2 \alpha V
$$

it is preserved during further expansion. The kinetic part of the scalar field is transforming into matter, presumably dark matter, and the constant $\alpha$ determines the ratio of the the dark energy density, represented by $V$, to the matter density, represented by the kinetic term. As follows from observations, the main part of DE is represented presently by DE, which may be considered as the Einstein constant $\Lambda$. At earlier times the input of constant $\Lambda$ is smaller than the input of $\Lambda_{V}$, for a wide interval of constant $\alpha$ values.

Let us consider an expanding flat universe, described by the Friedmann equation [7]

$$
\frac{\dot{a}^{2}}{a^{2}}=\frac{8 \pi G}{3} \rho+\frac{\Lambda}{3}
$$

Introduce

$$
\rho_{\phi}=V, \quad P_{\phi}=-V, \quad \rho_{m}=\frac{\dot{\phi}^{2}}{2}, \quad P_{m}=\beta \frac{\dot{\phi}^{2}}{2}, \quad \text { with } \quad \rho_{m}=\alpha \rho_{\phi} .
$$

We suggest that only part $\beta$ of the kinetic term makes the input into the pressure of the matter, so it follows from (3) and (5)

$$
\rho=\rho_{\phi}+\rho_{m}=(1+\alpha) V, \quad P=P_{\phi}+P_{m}=-(1-\alpha \beta) V .
$$

The adiabatic condition

$$
\frac{d \rho}{\rho+P}=-\frac{d \mathcal{V}}{\mathcal{V}}=-3 \frac{d a}{a}, \quad \mathcal{V} \text { is the volume, }
$$

may be written as

$$
\begin{gathered}
\dot{\rho}=-3 \frac{\dot{a}}{a}\left(\rho_{\phi}+\rho_{m}+P_{\phi}+P_{m}\right)=-3 \frac{\dot{a}}{a}\left(\rho_{m}+P_{m}\right)=-3 \alpha \frac{1+\beta}{1+\alpha} \frac{\dot{a}}{a} \rho . \\
\frac{\rho}{\rho_{*}}=\left(\frac{a}{a_{*}}\right)^{\frac{3 \alpha}{2(1+\alpha)}} \text { for } \beta=0 .
\end{gathered}
$$

\subsection{A Universe with $\Lambda=0$}

Suggest first that cosmological constant $\Lambda=0$, and DE is created only by the part of the scalar field $\Lambda_{V}$, represented by $V$. The expressions for the total density $\rho$, scaling factor $a$, and Hubble "constant" $H$ follow from (4)-(8) as

$$
\begin{aligned}
& \frac{a}{a_{*}}=\left(6 \pi G \rho_{*} t^{2}\right)^{\frac{1+\alpha}{3 \alpha(1+\beta)}}\left(\frac{\alpha(1+\beta)}{1+\alpha}\right)^{\frac{2(1+\alpha)}{3 \alpha(1+\beta)}}=\left(\frac{\rho_{*}}{\rho}\right)^{\frac{1+\alpha}{3 \alpha(1+\beta)}}=\left(\frac{t}{t_{*}}\right)^{\frac{2(1+\alpha)}{3 \alpha(1+\beta)}} \\
& \rho=\left(\frac{1+\alpha}{\alpha(1+\beta)}\right)^{2} \frac{1}{6 \pi G t^{2}}, \quad H=\frac{\dot{a}}{a}=\frac{2(1+\alpha)}{3 \alpha(1+\beta) t} .
\end{aligned}
$$

Here $\rho_{*}=\rho\left(t_{*}\right), a_{*}=a\left(t_{*}\right), t_{*}$ is an arbitrary time moment. Write the expressions for particular cases. For $\beta=1 / 3$ (radiation dominated universe) it follows from (10) 


$$
\begin{gathered}
\frac{a}{a_{*}}=\left(6 \pi G \rho_{*} t^{2}\right)^{\frac{1+\alpha}{4 \alpha}}\left[\frac{4 \alpha}{3(1+\alpha)}\right]^{\frac{1+\alpha}{2 \alpha}}=\left(\frac{\rho_{*}}{\rho}\right)^{\frac{1+\alpha}{4 \alpha}}=\left(\frac{t}{t_{*}}\right)^{\frac{1+\alpha}{2 \alpha}}, \\
\rho=\left(\frac{3(1+\alpha)}{4 \alpha}\right)^{2} \frac{1}{6 \pi G t^{2}}, \quad H=\frac{\dot{a}}{a}=\frac{1+\alpha}{2 \alpha t} .
\end{gathered}
$$

For the value of $\beta=0$ (dusty universe, $z<1100$ ) we have

$$
\begin{gathered}
\frac{a}{a_{*}}=\left(6 \pi G \rho_{*} t^{2}\right)^{\frac{1+\alpha}{3 \alpha}}\left[\frac{\alpha}{1+\alpha}\right]^{\frac{2(1+\alpha)}{3 \alpha}}=\left(\frac{\rho_{*}}{\rho}\right)^{\frac{1+\alpha}{3 \alpha}}=\left(\frac{t}{t_{*}}\right)^{\frac{2(1+\alpha)}{3 \alpha}}, \\
\rho=\left(\frac{1+\alpha}{\alpha}\right)^{2} \frac{1}{6 \pi G t^{2}}, \quad H=\frac{\dot{a}}{a}=\frac{2(1+\alpha)}{3 \alpha t} .
\end{gathered}
$$

\subsection{A Universe in the Presence of the Cosmological Constant $\Lambda$}

Equations (5)-(8) are valid in the presence of $\Lambda$. The solution of Equation (4) with nonzero $\Lambda$ is written in the form

$$
\begin{gathered}
\left(\frac{a}{a_{*}}\right)^{\frac{3 \alpha(1+\beta)}{2(1+\alpha)}}=\sqrt{\frac{8 \pi G \rho_{*}}{\Lambda c^{2}}} \sinh \left(\sqrt{\left.\frac{\Lambda}{3} \frac{3 \alpha(1+\beta)}{2(1+\alpha)} c t\right)=\sqrt{\frac{\rho_{*}}{\rho}}}\right. \\
\sqrt{\frac{\Lambda c^{2}}{8 \pi G \rho}}=\sinh \left(\sqrt{\frac{\Lambda}{3}} \frac{3 \alpha(1+\beta)}{2(1+\alpha)} c t\right), \quad H=\frac{\dot{a}}{a}=\sqrt{\frac{\Lambda c^{2}}{3}} \operatorname{coth}\left(\sqrt{\left.\frac{\Lambda}{3} \frac{3 \alpha(1+\beta)}{2(1+\alpha)} c t\right) .}\right.
\end{gathered}
$$

For the dusty universe $(\beta=0)$, after recombination at $z<1100$ we have

$$
\begin{array}{r}
\left(\frac{a}{a_{*}}\right)^{\frac{3 \alpha}{2(1+\alpha)}}=\sqrt{\frac{8 \pi G \rho_{*}}{\Lambda c^{2}}} \sinh \left(\sqrt{\frac{\Lambda}{3}} \frac{3 \alpha}{2(1+\alpha)} c t\right)=\sqrt{\frac{\rho_{*}}{\rho}} ; \\
\sqrt{\frac{\Lambda c^{2}}{8 \pi G \rho}}=\sinh \left(\sqrt{\frac{\Lambda}{3}} \frac{3 \alpha}{2(1+\alpha)} c t\right), \quad H=\frac{\dot{a}}{a}=\sqrt{\frac{\Lambda c^{2}}{3}} \operatorname{coth}\left(\sqrt{\frac{\Lambda}{3}} \frac{3 \alpha}{2(1+\alpha)} c t\right) .
\end{array}
$$

The dusty universe without DEV is described by relations following from (15) and (16) at $\alpha \rightarrow \infty$, giving

$$
\begin{gathered}
\left(\frac{a}{a_{*}}\right)^{\frac{3}{2}}=\sqrt{\frac{8 \pi G \rho_{*}}{\Lambda c^{2}}} \sinh \left(\sqrt{\frac{\Lambda}{3}} \frac{3}{2} c t\right)=\sqrt{\frac{\rho_{*}}{\rho}} \\
\sqrt{\frac{\Lambda c^{2}}{8 \pi G \rho}}=\sinh \left(\sqrt{\frac{\Lambda}{3}} \frac{3}{2} c t\right), \quad H=\frac{\dot{a}}{a}=\sqrt{\frac{\Lambda c^{2}}{3}} \operatorname{coth}\left(\sqrt{\frac{\Lambda}{3}} \frac{3}{2} c t\right) .
\end{gathered}
$$

\section{Hubble Tension}

Recently, a challenge in cosmology was formulated because of different values, obtained from different experiments, of the Hubble constant at the present epoch. There is a significant discrepancy (tension) between the Planck measurement from cosmic microwave background (CMB) anisotropy, where the best-fit model gives [14-16],

$$
H_{0}^{\mathrm{P} 18}=67.36 \pm 0.54 \mathrm{~km} \mathrm{~s}^{-1} \mathrm{Mpc}^{-1},
$$

and measurements using type Ia supernovae (SNIa) calibrated with Cepheid distances [17-21],

$$
H_{0}^{\mathrm{R} 19}=74.03 \pm 1.42 \mathrm{~km} \mathrm{~s}^{-1} \mathrm{Mpc}^{-1} .
$$

Measurements using time delays from lensed quasars [22] gave the value $H_{0}=73.3_{-1.8}^{+1.7} \mathrm{~km} \mathrm{~s}^{-1} \mathrm{Mpc}^{-1}$, while in [23] it was found that 
$H_{0}=72.4 \pm 1.9 \mathrm{~km} \mathrm{~s}^{-1} \mathrm{Mpc}^{-1}$ using the tip of the red giant branch applied to SNIa, which is independent of the Cepheid distance scale. Analysis of a compilation of these and other recent high- and low-redshift measurements shows [24] that the discrepancy between Planck [16] and any three independent late-Universe measurements is between $4 \sigma$ and $6 \sigma$. Different sophisticated explanations for the appearance of HT have been proposed [25-30] (see also [31-34]) and new experiments have been proposed for checking the reliability of this tension [35] (see also review [36]).

Dark matter (DM) and dark energy (DE) represent about $96 \%$ of the universe constituents $[14,17,18]$, but their origin is still not clear. The present value of DE density may be represented by the Einstein cosmological constant $\Lambda$ [37], but may also be a result of the action of the Higgs-type scalar fields, which are supposed to be the reason for the inflation in the early universe [10] (see also [11,12,38]). The value of the induced $\Lambda$, suggested for the inflation, is many orders of magnitude larger than its present value, and no attempts have been made to find a connection between them. The origin of DM is even more vague. There are numerous suggestions for its origin [39-41], but none of these possibilities has been experimentally or observationally confirmed, while many of them have been disproved.

To explain the origin of the Hubble Tension, we introduce a variable part of the cosmological "constant" $\Lambda_{V}$, proportional to the matter density $\rho_{D M}=\alpha \rho_{D E V}$. This part of $\Lambda_{V}$ influences the cosmological expansion at large redshifts, where the influence of the real Einstein constant $\Lambda$ is negligible. The value of $\Lambda_{V}$ is represented by a small component of $\mathrm{DE}$, which we define as DEV. We suppose here, without knowledge of the physical properties of DM particles, that there is a wide spectrum of DM particle, which could be produced by DEV until present time.

This seems necessary because at decreasing of the DEV field strength in the expanding universe it would be able to make mutual transformations only with DM particles of decreasing mass. The existence of particles with a very low rest mass (axions [42]) is considered often as a candidate for DM. Note that in the paper of Yu N. Gnedin [5], mutual transformations between axions and electromagnetic photons have been considered, instead of the hypothetical "scalar field" in our model.

\section{Removing the Hubble Tension}

We consider a model of the expanding universe after recombination, at $z \leq 1100$, with a fixed ratio $\alpha$ of energy densities between DEV, connected with a scalar field, cosmological constant $\Lambda$, and DM. If the mass spectrum of DM particles prolongs to very small masses, then we may expect an almost constant DEV-DM ratio. In the inflation model of the universe, only a scalar field was born at the very beginning, and matter was created in the process of expansion from the dynamic part of the scalar field density.

Here we show that in presence of DEV the Hubble value $H$ is decreasing with time slower than without it. This creates a larger present value of $H_{0}$, removing the Hubble tension at $\alpha \sim 140$. The main idea of removing the tension is the following. The CMB measurements give the value of the Hubble constant $H_{r}$, at the redshift $z \sim 1100$, close to the moment of recombination. This value is used for calculation of the present value of $H_{0}$.

For analysis of the Hubble tension it is more convenient to use logarithmic variables, so that from (10), (19) and (20) we have

$$
\begin{gathered}
\log H_{0}^{P 18}=\log 67.36=1.83 ; \quad \log H_{0}^{R 19}=\log 74.03=1.87 ; \\
\log \frac{H_{0}^{R 19}}{H_{0}^{P 18}}=\Delta \log H_{0}=0.04 .
\end{gathered}
$$

The Planck value $H_{r}^{P}$ was measured at the moment of recombination $z_{r} \approx 1100$, and extrapolated to present time using a dusty flat Friedmann model with a very small input from the cosmological constant $\Lambda$, when the values $z, \omega, a$ are connected as [7]

$$
z+1=\frac{\omega}{\omega_{0}}=\frac{a_{0}}{a} .
$$


In the case of an equipartition universe the extrapolation should be performed using Equation (16). Numerical modeling of large-scale structure formation gives preference to the cold dark matter model, corresponding to $P_{m} \approx 0, \beta=0$. In our interpretation the Hubble tension is connected with incorrect extrapolation by Equation (16) without DEV. From the value of Hubble tension in Equation (21) we may estimate $\alpha$ from the condition that both measurements are correct, but the reported value $H_{0}^{P 18}$ is coming from the incorrect extrapolation, and the actual present epoch value of the Hubble constant is determined by $H_{0}^{R 19}$.

From Equations (16) and (22) we obtain for a dusty universe, at $\beta=0$, the following connection between the recombination redshift $z_{r} \approx 1100$, the present age of the universe $t_{0}$ and the age of the universe $t_{r}$, corresponding to the recombination, in the form

$$
\begin{gathered}
z_{r \alpha}+1=\frac{a_{*}}{a_{r \alpha}}=\left[\sqrt{\frac{8 \pi G \rho_{*}}{\Lambda c^{2}}} \sinh \left(\sqrt{\frac{\Lambda}{3}} \frac{3 \alpha}{2(1+\alpha)} c t_{r \alpha}\right)\right]^{-\frac{2(1+\alpha)}{3 \alpha}}=\left(\frac{\rho_{*}}{\rho_{r \alpha}}\right)^{-\frac{(1+\alpha)}{3 \alpha}}, \\
H=\frac{\dot{a}}{a}=\sqrt{\frac{\Lambda c^{2}}{3}} \operatorname{coth}\left(\sqrt{\frac{\Lambda}{3}} \frac{3 \alpha}{2(1+\alpha)} c t_{r \alpha}\right) .
\end{gathered}
$$

Here indices with $\alpha$ indicate the values in the universe with DEV. The index " 0 " is related to present time at $z_{0}=0$. The index " $r$ " is related to the moment of recombination. The values in the universe without DEV do not contain $\alpha$ in the indices, and are written as

$$
\begin{gathered}
z_{r}+1=\frac{a_{*}}{a_{r}}=\left[\sqrt{\frac{8 \pi G \rho_{*}}{\Lambda c^{2}}} \sinh \left(\sqrt{\frac{\Lambda}{3}} \frac{3}{2} c t_{r}\right)\right]^{-\frac{2}{3}}=\left(\frac{\rho_{*}}{\rho_{r}}\right)^{-\frac{1}{3}}, \\
H=\frac{\dot{a}}{a}=\sqrt{\frac{\Lambda c^{2}}{3}} \operatorname{coth}\left(\sqrt{\frac{\Lambda}{3}} \frac{3}{2} c t_{r}\right) .
\end{gathered}
$$

The observational data are connected with a redshift, so we find the expression for the time as a function of the redshift in the form

$$
\begin{gathered}
c t_{r \alpha}=\frac{2(1+\alpha)}{3 \alpha} \sqrt{\frac{3}{\Lambda}} \sinh ^{-1}\left[\sqrt{\frac{\Lambda c^{2}}{8 \pi G \rho_{*}}}\left(z_{r \alpha}+1\right)^{\frac{3 \alpha}{2(1+\alpha)}}\right] \\
\approx \frac{\alpha+1}{\alpha \sqrt{6 \pi G \rho_{*}}}\left(z_{r \alpha}+1\right)^{\frac{3 \alpha}{2(1+\alpha)}}, \\
c t_{0 \alpha}=\frac{2(1+\alpha)}{3 \alpha} \sqrt{\frac{3}{\Lambda}} \sinh ^{-1}\left(\sqrt{\frac{\Lambda c^{2}}{8 \pi G \rho_{*}}}\right) .
\end{gathered}
$$

At $t=t_{r \alpha}$ the argument of "sinh" is very small, so only the first term in the expansion remains. Corresponding values for the universe without DEV are written as

$$
\begin{gathered}
c t_{r}=\frac{2}{3} \sqrt{\frac{3}{\Lambda}} \sinh ^{-1}\left[\sqrt{\frac{\Lambda c^{2}}{8 \pi G \rho_{*}}}\left(z_{r}+1\right)^{\frac{3}{2}}\right] \approx \frac{1}{\sqrt{6 \pi G \rho_{*}}}\left(z_{r}+1\right)^{\frac{3}{2}}, \\
c t_{0 \alpha}=\frac{2(1+\alpha)}{3 \alpha} \sqrt{\frac{3}{\Lambda}} \sinh ^{-1}\left(\sqrt{\frac{\Lambda c^{2}}{8 \pi G \rho_{*}}}\right) .
\end{gathered}
$$

For $z_{r \alpha}=z_{r} \equiv z_{r e c}$ we obtain a connection between $t_{r \alpha}$ and $t_{r}$ as

$$
t_{r \alpha}=\frac{\alpha+1}{\alpha}\left(z_{r e c}+1\right)^{\frac{3}{2(1+\alpha)}} t_{r} .
$$

The ratio of Hubble constants at present time $t_{0}$ to its value at the recombination time $t_{r \alpha}$, in the presence of DEV, using (16), is written as 


$$
\frac{H_{0 \alpha}}{H_{r \alpha}}=\frac{\operatorname{coth}\left(\sqrt{\frac{\Lambda}{3}} \frac{3 \alpha}{2(1+\alpha)} c t_{0}\right)}{\operatorname{coth}\left(\sqrt{\frac{\Lambda}{3}} \frac{3 \alpha c t_{r \alpha}}{2(1+\alpha)}\right)}=\frac{\tanh \left(\sqrt{\frac{\Lambda}{3}} \frac{3 \alpha c t_{r \alpha}}{2(1+\alpha)}\right)}{\tanh \left(\sqrt{\frac{\Lambda}{3}} \frac{3 \alpha}{2(1+\alpha)} c t_{0}\right)}=\frac{\sqrt{\frac{\Lambda}{3}} \frac{3 \alpha}{2(1+\alpha)} c t_{r \alpha}}{\tanh \left(\sqrt{\frac{\Lambda}{3}} \frac{3 \alpha}{2(1+\alpha)} c t_{0}\right)} .
$$

The corresponding values for the case without DEV, at $\alpha \rightarrow \infty$, with recombination time $t_{r}$ are written as

$$
\frac{H_{0}}{H_{r}}=\frac{\operatorname{coth}\left(\sqrt{\frac{\Lambda}{3}} \frac{3}{2} c t_{0}\right)}{\operatorname{coth}\left(\sqrt{\frac{\Lambda}{3}} \frac{3 c t_{r}}{2}\right)}=\frac{\tanh \left(\sqrt{\frac{\Lambda}{3}} \frac{3 c t_{r}}{2}\right)}{\tanh \left(\sqrt{\frac{\Lambda}{3}} \frac{3}{2} c t_{0}\right)}=\frac{\sqrt{\frac{\Lambda}{3}} \frac{3}{2} c t_{r}}{\tanh \left(\sqrt{\frac{\Lambda}{3}} \frac{3}{2} c t_{0}\right)} .
$$

Using Equations (31)-(33), we obtain the ratio between the correct value $H_{0 \alpha}$, identified with the local measurement of $H$, and the value $H_{0}$, obtained by calculations without account of DEV, in the form

$$
\frac{H_{0 \alpha}}{H_{0}}=\frac{\tanh \left(\sqrt{\frac{\Lambda}{3}} \frac{3}{2} c t_{0}\right)}{\tanh \left(\sqrt{\frac{\Lambda}{3}} \frac{3 \alpha}{2(1+\alpha)} c t_{0}\right)}\left(z_{r e c}+1\right)^{\frac{3}{2(1+\alpha)}}
$$

\section{Numerical Estimations}

Let us consider the following commonly accepted, averaged parameters of the universe, used in relation to the HT explanation.

Local Hubble constant $H_{l}=73 \mathrm{~km} / \mathrm{s} / \mathrm{Mpc}$.

Distantly measured Hubble constant $H_{d}=67.5 \mathrm{~km} / \mathrm{s} / \mathrm{Mpc}$.

Total density of the flat universe $\rho_{t o t}=2 \cdot 10^{-29} h^{2}=1.066 \cdot 10^{-29} \mathrm{~g} / \mathrm{cm}^{3}$, where $\mathrm{h}=\mathrm{H} / 100 \mathrm{~km} / \mathrm{s} / \mathrm{Mpc}$, with $h=0.73$.

Distantly measured densities of the universe components, $\rho_{\Lambda_{*}}=0.7 \rho_{t o t}=7.5 \cdot 10^{-30} \mathrm{~g} / \mathrm{cm}^{3}$; $\rho_{m *}=0.3 \rho_{\text {tot }}$.

Locally measured cosmological constant density [18] $\rho_{\Lambda_{0}}=(0.44 \div 0.96) \rho_{t o t},(2 \sigma$ statistics).

$\Lambda=\frac{8 \pi G \rho_{\Lambda_{*}}}{c^{2}}=1.40 \cdot 10^{-56} \mathrm{~cm}^{-2}$.

Average age of the universe $t_{0}=4.35 \cdot 10^{17} \mathrm{~s}$ [43].

The ratio of two Hubble constants is $H T_{r}=\frac{H_{l}}{H_{d}}=1.08$.

Identifying $H_{0 \alpha} \equiv H_{l}, H_{0} \equiv H_{d}$, we obtain from (34)

$$
\begin{gathered}
\frac{H_{0 \alpha}}{H_{0}}=1.08=\frac{\tanh \left(\sqrt{\frac{\Lambda}{3}} \frac{3}{2} c t_{0}\right)}{\tanh \left(\sqrt{\frac{\Lambda}{3}} \frac{3 \alpha}{2(1+\alpha)} c t_{0}\right)}\left(z_{r e c}+1\right)^{\frac{3}{2(1+\alpha)}} \\
=\frac{\tanh (1.337)}{\tanh \left(1.337 \frac{\alpha}{1+\alpha}\right)}(1101)^{\frac{3}{2(1+\alpha)}}=\frac{0.87095}{\tanh \left(1.337 \frac{\alpha}{1+\alpha}\right)}(1101)^{\frac{3}{2(1+\alpha)}} .
\end{gathered}
$$

Finally we obtain the equation for $\alpha$ in the form

$$
\tanh \left(1.337 \frac{\alpha}{1+\alpha}\right)=0.8064(1101)^{\frac{3}{2(1+\alpha)}}, \quad \alpha \approx 140 .
$$

Taking $\rho_{m r}=0.3 \rho_{t o t}$, we obtain $\rho_{D E V}=0.0022 \rho_{t o t}$, and the effective dark energy density at present time should be equal to $\Omega_{0 e f f}=0.7022$, which is inside the limits of local measurement of $\Lambda_{0}$, as mentioned above. The local matter density of the flat universe at present time is $\Omega_{0 m}=0.2978$, leading to the dark matter density $\Omega_{0 d m}=0.2578$, for the baryon density $\Omega_{0 b}=0.04$. 


\section{Discussion}

We consider a cosmological model, where the DE input consists of two components: cosmological constant $\Lambda$, and small variable part DEV, which is connected with a scalar field, originated in the early universe and creating inflation. The energy density of this variable part is uniquely connected with a matter density, and the matter itself was created during the inflation stage. For simplicity we consider a cosmological model with a linear connection between energy densities as $\alpha \rho_{D E V}=\rho_{D M}$. This model could give a solution to the problem of Hubble Tension, which appears by using an inappropriate extrapolation when finding the present value of the Hubble constant from Planck observational data. We have solved the Friedmann equation in the presence of this relation, and have found the value of $\alpha$ at which HT disappeared. The present DEV density needed for explanation of HT phenomena is very small relative to the cosmological constant $\Lambda$. It influences the cosmological expansion at larger redshifts, where the input of the Einstein constant $\Lambda$ is small. Presently the situation is opposite, $\Lambda \gg \Lambda_{V}$, because decreasing of matter density during cosmological expansion determines the transition from the quasi-Friedmann to quasi-de Sitter stage. The estimation of the density $\rho_{\Lambda_{V}}$ at the present epoch corresponds to $\Omega_{\Lambda_{V}} \approx 0.0022$, which slightly increases the present dark energy density. In the flat universe it determines decreasing of the dark matter density due to transfer of its energy into DEV in the condition of "equipartition" at constant $\alpha$.

We have used for estimations a constant ratio of $\rho_{\Lambda_{V}} / \rho_{m}=1 / \alpha$ for the universe expansion after recombination, at $z<1100$, but deviations from this law should not qualitatively change the conclusion that a relatively small average contribution of the variable $\Omega_{\Lambda_{V}}$ may explain the difference in Hubble constant measured at local and high- $z$ distances.

The present parameters of the LCDM model have been estimated from the analysis of CMB fluctuation measurements in WMAP and PLANCK experiments, having a powerlaw spectrum of adiabatic scalar perturbations. The procedure is based on a search of extremes in the multidimensional parameter space. The presence of HT (if real) adds an additional restriction to this problem. The universe parameters obtained in this process may be changed with this additional restriction. The computations could be performed in the presence of a variable $\alpha$. Decreasing of dark matter leads to decreasing of the field amplitude, which may prevent the energy exchange between DM and DEV in the absence of very light DM particles.

In our model the DM should be represented by wide mass spectrum particles, and not by unique mass CDM particles, which are usually considered now. By analogy with $\mathrm{CMB}$, the lowest mass of DM particles should not presently exceed the value $\sim$ $\left(\Omega_{D E V} / \Omega_{C M B}\right)^{1 / 4} \times k T_{C M B} \approx 7 \cdot 10^{-4} \mathrm{eV}$, to retain the possibility of an almost constant $\alpha$.

Funding: This work was supported by the Russian Science Foundation (grant No. 18-12-00378).

Acknowledgments: The author is very grateful to O. Yu. Tsupko for valuable comments.

Conflicts of Interest: The author declares no conflict of interest.

\section{Note}

In most equations below it is taken that $c=1$.

\section{References}

1. Gnedin, Y.N.; Krasnikov, S.V. Polarimetric effects associated with the detection of Goldstone bosons in stars and galaxies. Sov. Phys. JETP 1992, 75, 933-937.

2. Gnedin, Y.N. Resonance magnetic conversion of photons into massless axions and striking feature in quasar polarized light. Astrophys. Space Sci. 1997, 249, 125-149. [CrossRef]

3. Gnedin, Y.N. Astronomical searches for nonbarionic dark matter. Astrophys. Space Sci. 1997, 252, 95-106. [CrossRef]

4. Gnedin, Y.N.; Dodonov, S.N.; Vlasyuk, V.V.; Spiridonova, O.I.; Shakhverdov, A.V. Astronomical searches for axions: Observations at the SAO 6-m telescope. Mon. Not. R. Astron. Soc. 1999, 306, 117-121. [CrossRef]

5. Gnedin, Y.N.; Piotrovich, M.Y.; Natsvlishvili, T.M. PVLAS experiment: Some astrophysical consequences. Mon. Not. R. Astron. Soc. 2007, 374, 276-281. [CrossRef] 
6. Piotrovich, M.Y.; Gnedin, Y.N.; Natsvlishvili, T.M. Coupling constant for axion and electromagnetic fields and cosmological observations. Astrophysics 2009, 52, 412-422. [CrossRef]

7. Zel'dovich, Y.B.; Novikov, I.D. The Structure and Evolution of the Universe; Nauka: Moscow, Russia, 1975.

8. Riess, A.G. The Expansion of the Universe is Faster than Expected. Nat. Rev. Phys. 2019, 2, 10-12. [CrossRef]

9. Freedman, W.L. Measurements of the Hubble Constant: Tensions in Perspective. Astrophys. J. 2021, 919, 16. [CrossRef]

10. Guth, A.H. The Inflationary Universe; Perseus Books: Reading, MA, USA, 1998.

11. Linde, A.D. Chaotic inflation. Phys. Lett. B 1983, 129, 177-181. [CrossRef]

12. Starobinsky, A.A. Dynamics of phase transition in the new inflationary universe scenario and generation of perturbations. Phys. Lett. B 1982, 117, 175-178. [CrossRef]

13. Peebles, P.J.E. Principles of Physical Cosmology; Princeton University Press: Princeton, NJ, USA, 1993.

14. Spergel, D.N.; Verde, L.; Peiris, H.V.; Komatsu, E.; Nolta, M.R.; Bennett, C.L.; Halpern, M.; Hinshaw, G.; Jarosik, N.; Kogut, A.; et al. First-Year Wilkinson Microwave Anisotropy Probe (WMAP) Observations: Determination of Cosmological Parameters. Astrophys. J. Suppl. Ser. 2003, 148, 175-194. [CrossRef]

15. Ade, P.A.R.; Aghanim, N.; Arnaud, M.; Ashdown, M.; Aumont, J.; Baccigalupi, C.; Banday, A.J.; Barreiro, R.B.; Bartlett, J.G.; Bartolo, N.; et al. Planck Collaboration. Planck 2015 results. XIII. Cosmological parameters. Astron. Astrophys. 2016, 594, A13.

16. Aghanim, N.; Akrami, Y.; Ashdown, M.; Aumont, J.; Baccigalupi, C.; Ballardini, M.; Banday, A.J.; Barreiro, R.B.; Bartolo, N.; Basak, S.; et al. Planck Collaboration. Planck 2018 results. VI. Cosmological parameters. arXiv 2020, arXiv:1807.06209v3.

17. Riess, A.G.; Filippenko, A.V.; Challis, P.; Clocchiatti, A.; Diercks, A.; Garnavich, P.M.; Gilliland, R.L.; Hogan, C.J.; Jha, S.; Kirshner, R.P.; et al. Observational Evidence from Supernovae for an Accelerating Universe and a Cosmological Constant. Astron. J. 1998, 116, 1009-1038. [CrossRef]

18. Perlmutter, S.; Aldering, G.; Goldhaber, G.; Knop, R.A.; Nugent, P.; Castro, P.G.; Deustua, S.; Fabbro, S.; Goobar, A.; Groom, D.E.; et al. Measurements of $\Omega$ and $\Lambda$ from 42 High-Redshift Supernovae. Astrophys. J. 1999, 517, 565-586. [CrossRef]

19. Riess, A.G.; Macri, L.M.; Hoffmann, S.L.; Scolnic, D.; Casertano, S.; Filippenko, A.V.; Tucker, B.E.; Reid, M.J.; Jones, D.O.; Silverman, J.M.; et al. A 2.4\% Determination of the Local Value of the Hubble Constant. Astrophys. J. 2016, 826, 56. [CrossRef]

20. Riess, A.G.; Casertano, S.; Yuan, W.; Macri, L.; Bucciarelli, B.; Lattanzi, M.G.; MacKenty, J.W.; Bowers, J.B.; Zheng, W.; Filippenko, A.V.; et al. Milky Way Cepheid Standards for Measuring Cosmic Distances and Application to Gaia DR2: Implications for the Hubble Constant. Astrophys. J. 2018, 861, 126. [CrossRef]

21. Riess, A.G.; Casertano, S.; Yuan, W.; Macri, L.M.; Scolnic, D. Large Magellanic Cloud Cepheid Standards Provide a 1\% Foundation for the Determination of the Hubble Constant and Stronger Evidence for Physics beyond $\Lambda$ CDM. Astrophys. J. 2019, 876, 85. [CrossRef]

22. Wong, K.C.; Suyu, S.H.; Chen, G.C.; Rusu, C.E.; Millon, M.; Sluse, D.; Bonvin, V.; Fassnacht, C.D.; Taubenberger, S.; Auger, M.W.; et al. H0LiCOW XIII. A $2.4 \%$ measurement of $H_{0}$ from lensed quasars: $5.3 \sigma$ tension between early and late-Universe probes. Mon. Not. R. Astron. Soc. 2020, 498, 1420-1439. [CrossRef]

23. Yuan, W.; Riess, A.G.; Macri, L.M.; Casertano, S.; Scolnic, D.M. Consistent Calibration of the Tip of the Red Giant Branch in the Large Magellanic Cloud on the Hubble Space Telescope Photometric System and Implications for the Determination of the Hubble Constant. Astrophys. J. 2019, 886, 61. [CrossRef]

24. Verde, L.; Treu, T.; Riess, A.G. Tensions between the Early and the Late Universe. arXiv 2019, arXiv:1907.10625.

25. Karwal, T.; Kamionkowski, M. Early dark energy, the Hubble-parameter tension, and the string axiverse. Phys. Rev. D 2016, 94, 103523. [CrossRef]

26. Moertsell, E.; Dhawan, S. Does the Hubble constant tension call for new physics? arXiv 2018, arXiv:1801.07260.

27. Poulin, V.; Smith, T.L.; Karwal, T.; Kamionkowski, M. Early Dark Energy Can Resolve The Hubble Tension. Phys. Rev. Lett. 2019, 122, 221301. [CrossRef]

28. Yang, W.; Pan, S.; Di Valentino, E.; Nunes, R.C.; Vagnozzi, S.; Mota, D.F. Tale of stable interacting dark energy, observational signatures, and the H0 tension. J. Cosmol. Astropart. Phys. 2018, 1809, 019. [CrossRef]

29. Vagnozzi, S. New physics in light of the H0 tension: An alternative view. arXiv 2019, arXiv:1907.07569.

30. Di Valentino, E.; Melchiorri, A.; Mena, O.; Vagnozzi, S. Interacting dark energy after the latest Planck, DES, and H0 measurements: An excellent solution to the $\mathrm{H} 0$ and cosmic shear tensions. arXiv 2019, arXiv:1908.04281.

31. Umiltà, C.; Ballardini, M.; Finelli, F.; Paoletti, D. CMB and BAO constraints for an induced gravity dark energy model with a quartic potential. J. Cosmol. Astropart. Phys. 2015, 1508, 017. [CrossRef]

32. Ballardini, M.; Finelli, F.; Umiltà, C.; Paoletti, D. Cosmological constraints on induced gravity dark energy models. J. Cosmol. Astropart. Phys. 2016, 1605, 067. [CrossRef]

33. Rossi, M.; Ballardini, M.; Braglia, M.; Finelli, F.; Paoletti, D.; Starobinsky, A.A.; Umilta, C. Cosmological constraints on postNewtonian parameters in effectively massless scalar-tensor theories of gravity. Phys. Rev. D 2019, 100, 103524. [CrossRef]

34. Knox, L.; Millea, M. The Hubble Hunter's Guide. Phys. Rev. D 2020, 101, 043533. [CrossRef]

35. Bengaly, C.A.P.; Clarkson, C.; Maartens, R. The Hubble constant tension with next generation galaxy surveys. arXiv 2019, arXiv:1908.04619.

36. Di Valentino, E.; Mena, O.; Pan, S.; Visinelli, L.; Yang, W.; Melchiorri, A.; Mota, D.F.; Riess, A.G.; Silk, J. In the realm of the Hubble tension-A review of solutions. Class. Quantum Grav. 2021, 38, 153001. [CrossRef] 
37. Einstein, A. Kosmologische Betrachtungen zur allgemeinen Relativitätstheorie. In Sitzungsberichte der Königlich Preussischen Akademie der Wissenschaften Berlin-Part 1; 1917; pp. 142-152.

38. Mukhanov, V.F.; Chibisov, G.V. Vacuum energy and large-scale structure of the Universe. Sov. Phys. JETP 1982, 56, $258-265$.

39. Arun, K.; Gudennavar, S.B.; Sivaram, C. Dark matter, dark energy, and alternate models: A review. Adv. Space Res. 2017, 60, 166-186. [CrossRef]

40. Zhao, G.B.; Raveri, M.; Pogosian, L.; Wang, Y.; Crittenden, R.G.; Handley, W.J.; Percival, W.J.; Beutler, F.; Brinkmann, J.; Chuang, C.H.; et al. Dynamical dark energy in light of the latest observations. Nat. Astron. 2017, 1, 627-632. [CrossRef]

41. Samart, D.; Phongpichit, P. Unification of inflation and dark matter in the Higgs-Starobinsky model. Eur. Phys. J. C 2019, 79, 347. [CrossRef]

42. Turner, M.S. Windows on the axion. Phys. Rep. 1990, 197, 67-97. [CrossRef]

43. Age of the Universe. Available online: https:/ / en.wikipedia.org/wiki/Age_of_the_universe (accessed on 28 October 2021). 